\title{
Use of Chinese medicine correlates negatively with the consumption of conventional medicine and medical cost in patients with uterine fibroids: a population-based retrospective cohort study in Taiwan
}

\author{
Shan-Yu Su ${ }^{1,2^{*}}$, Chih-Hsin Muo ${ }^{3,4}$ and Donald E Morisky ${ }^{5}$
}

\begin{abstract}
Background: Chinese medicine is commonly used and covered by health insurance to treat symptoms of uterine fibroids in Taiwan. This retrospective cohort study compared the consumption of conventional western medicine and medical cost between Chinese medicine (CM) users and nonusers among patients with uterine fibroids.

Methods: We extracted 44,122 patients diagnosed with uterine fibrosis between 1996 and 2010 from the National Health Insurance reimbursement database, which is a population-based database released by a government-run health insurance system. Multivariate linear regression models were used to find association between using Chinese medicine and the consumption of conventional medicine, and between using Chinese medicine and medical cost.

Results: The total fibroid-related conventional western medicine consumed by CM users was less than that by nonusers $(\beta=-10.49, P<0.0001)$. Three categories of conventional medicines, including antianemics $(-3.50$ days/year/patient, $P<0.0001)$, hemostatics (- 1.89 days/year/patient, $P<0.0001)$, and hormone-related agents $(-3.13$ days/year/patient, $P<0.0001$ ), were used less in patients who were CM users. Moreover, although using CM increased 16.9 USD per patient in $C M$ users annually $(P<0.0001)$, the total annual medical cost for treating fibroid was 5610 USD less in CM users than in nonusers $(P<0.0001)$.
\end{abstract}

Conclusions: Our results suggested that $\mathrm{CM}$ reduced the consumption of conventional medicine, and might be a potential therapeutic substitute for conventional western medicines to treat uterine fibroids with low cost.

Keywords: Uterine fibroid, Chinese medicine, Conventional western medicine, Iron-deficiency anemia, Hypermenorrhea, Dysmenorrhea

\section{Background}

Uterine fibroids are the most common benign uterine tumors, which happens in $60 \%$ of reproductive aged women [1]. The majority of fibroids are asymptomatic, while in $20 \%$ of patients it causes symptoms such as hypermenorrhea, dysmenorrhea, iron-deficiency anemia, and infertility [2]. In symptomatic patients, the first-line

\footnotetext{
* Correspondence: shanyusu@yahoo.com.tw

'Department of Chinese Medicine, China Medical University Hospital, No. 2

Yude road, Taichung 40447, Taiwan

${ }^{2}$ School of Post-baccalaureate Chinese Medicine, College of Chinese

Medicine, China Medical University, Taichung 40402, Taiwan

Full list of author information is available at the end of the article
}

treatment is surgery. However, since surgery often cause complications, conservative medical treatment to control symptoms related to fibroids are frequently adopted by patients and gynecologists [3]. Available indicated medical treatments include antianemics (blood derivatives and iron preparations) to treat iron-deficient anemia, hemostatics (coagulants and anti-fibrinolytic agents) to reduce bleedings of hypermenorrhea, and non-steroid anti-inflammatory drugs (NSAIDs) to relief pain. As no currently validated medical treatment is capable of making fibroid disappear, there are no reason to consider medical treatment in case of asymptomatic fibroids [4].

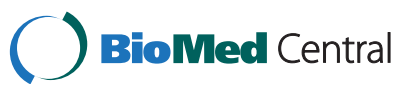


The estimated direct cost related to uterine fibroids includes cost of surgery, hospital admission, outpatient visit, and medication. The annual cost of uterine fibroids is up to 4.1-9.4 billion US dollars (USD) in the United States [5,6], including 3.27 to 5.10 billion USD in nonsurgical management and 829 million to 4.3 billion USD annually in surgical management [5]. If the indirect cost, including lost work-hour costs and obstetric outcomes cost, is taken into account, uterine fibroids are estimated to cost the United States 5.9 - 34.4 billion USD annually [5]. In European countries, the number of hospital admissions involving treatment for uterine fibroids ranges from 0.71 to 1.53 per 1000 women, and the estimated direct cost related to uterine fibroids ranges from two to five euros per woman [7].

Chinese medicine $(\mathrm{CM})$ is an alternative and complementary medicine to treat gynecological diseases in Asian countries [8-10]. Among fibroid patients, three fourth of them have the experience of utilizing CM, which are considered to be with fewer side effects, less expensive [11,12], less subsequent surgery rate [13], and higher life quality than conventional western medicine [14]. Because of the high usage rate, the expense of CM in Taiwan has been covered by the government-run National Health Insurance (NHI) system since 1995 [15]. Despite of the above-mentioned advantages, it is still unclear that whether use of CM decrease the consumption of conventional western medicine, in the case of that CM users usually have more symptoms than CM nonusers [13], and are supposed to use more medical resources. Moreover, as CM generates a new component of medical cost, whether the use of CM decreases the total medical cost also needed to be clarified.

In comparing fibroid patients who were CM users with CM nonusers, we sought to determine: (1) if the use of CM correlates with the consumption of conventional western medicines that is specifically for symptoms related to fibroids, and, (2) if the use of CM changes the Nation's health care cost for uterine fibroids.

\section{Methods}

\section{Subjects}

Patients with uterine fibroids were identified according to the International Classification of Diseases, $9^{\text {th }}$ Revision, Clinical Modification (ICD-9-CM) code 218 from 1996 to 2010 in the Longitudinal Health Insurance Database 2000 (LHID2000). The LHID2000 was set up by Taiwan's National Health Research Institute and it contains chronological information about one million randomly selected individuals who were beneficiaries from 1996 to 2000. This study was approved by the Research Ethics Committee of the CMUH (CMU-REC101-012).

\section{Study designs}

The date of the first diagnosis was used as the entry date, and the endpoint date was December 31th 2010, the date of death, withdrawal from the insurance program, or underwent uterine surgery. The follow-up time was defined as the period from the diagnosis date to the endpoint date. CM users were defined as subjects who had received a mean orally administered CM treatment for more than fifteen days per year. The total fibroid related consumption of western medicines was estimated by summarizing the number of days for which western medicines have been prescribed with a ICD-9-CM code of 218 from the diagnosis date to the endpoint date. Medical costs included insurance claim for the NHI reimbursement for outpatients (OPD), inpatients (IPD), emergency room (ER), and CM treatments that was related to uterine fibroid.

The examined variables included socio-demographic factors, including age $(<20,20-29,30-39,40-49$, and $>50$ years), income level $(<520,520-639,640-759$, and $\geq 759$ USD, based on quartile), and occupation status (white collar, blue collar, and others), and endometriosisrelated co-morbidities, including hypermenorrhea (ICD9-CM 626.2), iron-deficiency anemia (ICD-9-CM 280), dysmenorrhea (ICD-9-CM 625.3), and infertility (ICD9-CM 628 and 628.3).

\section{Statistical analysis}

In terms of categorical and ordinal variables, CM users and CM nonusers were compared using chi-square tests and Wilcoxon rank sum test, respectively. Multivariate linear regression models were performed to identify the independent factors associated to the consumption of western medicines, and to assess annual costs in OPD, IPD, ER, and $\mathrm{CM}$, as well as total annual medical cost. These adjusted models were controlled for age, occupation, income, and co-morbidities (hypermenorrhea, iron-deficiency anemia, dysmenorrhea, and infertility). Continuous data are expressed as mean \pm SD and categorical data are expressed as number and percentage. All statistical analyses were performed using SAS software, version 9.1 (SAS Institute Inc., Carey, NC), and the significance level was set at a two-tailed $P$ value of less than 0.05 .

\section{Results}

\section{Study subjects}

A total of 44,122 women diagnosed with uterine fibrosis between 1996 and 2010 were extracted from the LHID2000 database with a mean follow-up time of 4.6 years. Among them, 11,412 patients were identified as CM users, and 32,710 patients were identified as CM nonusers. The mean age of CM users (41.4 years) was younger than CM nonusers (42.4 years) (Table 1). The proportions of patients among CM users who were white collar, and registered 
Table 1 Comparison of socio-demographic factors and co-morbidities between Chinese medicine (CM) users and nonusers in patients with uterine fibroids

\begin{tabular}{|c|c|c|c|c|c|c|c|}
\hline & \multicolumn{4}{|l|}{$\mathrm{CM}$} & & & \multirow{4}{*}{$P$-value ${ }^{a}$} \\
\hline & \multirow{2}{*}{\multicolumn{2}{|c|}{$\begin{array}{l}\text { Nonusers } \\
\mathrm{N}=32710\end{array}$}} & \multirow{2}{*}{\multicolumn{2}{|c|}{$\begin{array}{l}\text { Users } \\
\mathrm{N}=11412\end{array}$}} & \multirow{2}{*}{\multicolumn{2}{|c|}{$\begin{array}{l}\text { Total } \\
\mathrm{N}=44122\end{array}$}} & \\
\hline & & & & & & & \\
\hline & $\mathrm{n}$ & $\%$ & $\mathrm{n}$ & $\%$ & $\mathrm{n}$ & $\%$ & \\
\hline Age, years & & & & & & & $<0.0001$ \\
\hline$<20$ & 202 & 0.6 & 47 & 0.4 & 249 & 0.5 & \\
\hline $20-29$ & 2634 & 8.1 & 1030 & 9.0 & 3664 & 8.3 & \\
\hline $30-39$ & 8998 & 27.5 & 3665 & 32.1 & 12663 & 28.7 & \\
\hline $40-49$ & 15559 & 47.6 & 5167 & 45.3 & 20726 & 47.0 & \\
\hline$\geq 50$ & 5317 & 16.5 & 1503 & 13.2 & 6820 & 15.5 & \\
\hline Mean \pm SD & $42.4 \pm$ & & $41.4 \pm$ & & $42.2 \pm$ & & \\
\hline Income, US\$ per month & & & & & & & 0.1800 \\
\hline$<520$ & 8075 & 24.7 & 2725 & 23.9 & 10800 & 24.5 & \\
\hline $520-639$ & 8173 & 25.0 & 2966 & 26.0 & 11139 & 25.3 & \\
\hline $640-759$ & 8556 & 26.2 & 2921 & 25.6 & 11477 & 26.0 & \\
\hline$\geq 760$ & 7906 & 24.2 & 2800 & 24.5 & 10706 & 24.3 & \\
\hline Mean \pm SD & 20098 & & 20333 & & 20158 & & \\
\hline Occupational status & & & & & & & $<0.0001$ \\
\hline White collar & 19243 & 58.8 & 6972 & 61.1 & 26215 & 59.4 & \\
\hline Blue collar & 9900 & 30.1 & 3312 & 29.0 & 13212 & 29.9 & \\
\hline Others & 3567 & 10.9 & 1128 & 9.9 & 4695 & 10.6 & \\
\hline Area & & & & & & & $<0.0001$ \\
\hline Northern Taiwan & 16281 & 50.0 & 4820 & 42.2 & 21101 & 47.8 & \\
\hline Central Taiwan & 5758 & 17.6 & 2656 & 23.3 & 8414 & 19.1 & \\
\hline Southern Taiwan & 9265 & 28.3 & 3511 & 30.8 & 12776 & 29.0 & \\
\hline Eastern Taiwan and offshore islands & 1406 & 4.3 & 425 & 3.7 & 1831 & 4.15 & \\
\hline Co-morbidity & & & & & & & \\
\hline Iron-deficiency anemia & 5259 & 16.1 & 2213 & 19.4 & 7472 & 16.9 & $<0.0001$ \\
\hline Hypermenorrhea & 8388 & 25.6 & 3650 & 32.0 & 12038 & 27.3 & $<0.0001$ \\
\hline Dysmenorrhea & 7456 & 22.8 & 4246 & 37.2 & 11702 & 26.5 & $<0.0001$ \\
\hline Infertility & 2570 & 7.9 & 1358 & 11.9 & 3928 & 8.9 & $<0.0001$ \\
\hline
\end{tabular}

${ }^{a}$ Chi-square test and Wilcoxon rank sum test.

their insurance in Central Taiwan were higher than that among CM nonusers. Moreover, the proportions of patients who were co-morbid with iron-deficiency anemia, hypermenorrhea, dysmenorrhea, and infertility were all higher among $\mathrm{CM}$ users than they were among CM nonusers.

Factors associated with the consumption of conventional western medicine

The mean number of days of fibroid-related western medicine taking was $14.6 \pm 50.6$ days per year. After adjusting for socio-demographic factors (age, income, occupation, and area), and co-morbid covariates (hypermenorrhea, iron-deficiency anemia, dysmenorrhea, and infertility) in a multivariate linear regression model, CM users consumed less western medicine than $\mathrm{CM}$ nonusers $(-10.5$ days/year/patient, $P<0.0001)$. Patients who aged higher than 40 years, who were blue collar, who registered their insurance in Central Taiwan, and whose income were less than $520 \mathrm{USD} /$ month tended to consume more conventional western medicine. Patients whose diseases were co-morbid with hypermenorrhea, iron-deficiency anemia (both $P<0.0001$ ), and dysmenorrhea $(P=0.0202)$ also consumed more western medicine than who were not co-morbid with these illnesses. However, patients whose diseases were co-morbid with infertility tended to consumed less western medicine than whose diseases were not co-morbid with infertility $(P=0.0295)$ (Table 2). 
Table 2 Multivariate linear regression for number of days of fibroid-related treatment in patients with uterine fibroid

\begin{tabular}{|c|c|c|c|c|c|}
\hline & Days/year $^{a}$ & Estimate $\beta$ & $\mathrm{VIF}^{\mathrm{b}}$ & $\mathbf{T}$ & $P$ \\
\hline \multicolumn{6}{|l|}{ CM } \\
\hline Nonusers & $17.2 \pm 56.8$ & - & - & - & - \\
\hline Users & $7.4 \pm 24.3$ & -10.49 & 1.03 & -19.03 & $<0.0001$ \\
\hline \multicolumn{6}{|l|}{ Age, years } \\
\hline$<20$ & $3.5 \pm 15.1$ & - & - & - & - \\
\hline $20-29$ & $6.1 \pm 27.3$ & 4.22 & 14.49 & 1.29 & 0.1971 \\
\hline $30-39$ & $11.0 \pm 41.3$ & 8.75 & 37.28 & 2.73 & 0.0063 \\
\hline $40-49$ & $18.4 \pm 58.3$ & 15.41 & 45.19 & 4.82 & $<0.0001$ \\
\hline$\geq 50$ & $15.0 \pm 50.6$ & 13.07 & 24.33 & 4.03 & $<0.0001$ \\
\hline \multicolumn{6}{|l|}{ Income, US\$ per month } \\
\hline$<520$ & $14.7 \pm 50.5$ & - & - & - & - \\
\hline $520-639$ & $13.9 \pm 48.7$ & -1.22 & 1.57 & -1.78 & 0.0745 \\
\hline 640- 759 & $17.1 \pm 56.2$ & -0.36 & 1.94 & -0.48 & 0.6300 \\
\hline$\geq 760$ & $12.8 \pm 45.9$ & -2.46 & 1.67 & -3.44 & 0.0006 \\
\hline \multicolumn{6}{|l|}{ Occupational status } \\
\hline White collar & $13.2 \pm 47.3$ & - & - & - & - \\
\hline Blue collar & $17.2 \pm 56.4$ & 1.70 & 1.48 & 2.70 & 0.007 \\
\hline Others & $15.1 \pm 50.9$ & 1.18 & 1.14 & 1.43 & 0.1514 \\
\hline \multicolumn{6}{|l|}{ Area } \\
\hline Northern Taiwan & $13.2 \pm 47.7$ & - & - & - & - \\
\hline Central Taiwan & $17.0 \pm 54.2$ & 3.57 & 1.16 & 5.48 & $<0.0001$ \\
\hline Southern Taiwan & $16.0 \pm 53.4$ & 2.25 & 1.20 & 3.92 & $<0.0001$ \\
\hline Eastern Taiwan and offshore islands & $12.4 \pm 44.4$ & -2.37 & 1.07 & -1.92 & 0.0543 \\
\hline \multicolumn{6}{|l|}{ Hypermenorrhea } \\
\hline No & $13.1 \pm 48.4$ & - & - & & - \\
\hline Yes & $18.8 \pm 55.8$ & 3.99 & 1.08 & 7.21 & $<0.0001$ \\
\hline \multicolumn{6}{|l|}{ Iron-deficiency anemia } \\
\hline No & $12.3 \pm 45.8$ & - & - & & - \\
\hline Yes & $26.2 \pm 68.4$ & 12.8 & 1.04 & 19.83 & $<0.0001$ \\
\hline \multicolumn{6}{|l|}{ Dysmenorrhea } \\
\hline No & $14.8 \pm 52.0$ & - & - & & - \\
\hline Yes & $14.2 \pm 46.3$ & 1.32 & 1.13 & 2.32 & 0.0202 \\
\hline \multicolumn{6}{|l|}{ Infertility } \\
\hline No & $15.2 \pm 51.7$ & - & - & & - \\
\hline Yes & $9.5 \pm 36.4$ & -1.88 & 1.08 & -2.18 & 0.0295 \\
\hline
\end{tabular}

${ }^{\mathrm{a}} \mathrm{R}$-square $=0.0277$ and adjusted R-square $=0.0274$ in a multivariate linear regression model that was adjusted for age, income, occupation, area, and iron-deficiency anemia, infertility, excessive bleeding, and dysmenorrhea.

${ }^{b}$ VIF, variance inflation factor.

Difference in the consumption of antianemics, hemostastics, analgesics, and hormone-related agents between CM users and nonusers

Taking all the subjects as a whole, multivariate linear regression revealed that $\mathrm{CM}$ users consumed less antianemics (-3.50 days/year/patient, $P<0.0001)$, hemostatics (- 1.89 days/year/patient, $P<0.0001)$, and hormone-related agents $(-3.13$ days/year/patient, $P<0.0001)$ than $C M$ nonusers (Table 3$)$. Stratification analysis showed that differences between CM users and nonusers in the consumption of antianemics substantially enlarged in patients with iron-deficiency anemia (-11.29 days/year/ patient, $P<0.0001$ ), that of hemostatics enlarged in patients with dysmenorrhea (-4.01 days/year/patient, $P<0.0001)$, 
Table 3 Consumption of antianemics, hemostatics, and analgesics in patients co-morbid with iron-deficiency anemia, hypermenorrhea, and dysmenorrhea

\begin{tabular}{|c|c|c|c|c|}
\hline & \multirow{2}{*}{$\begin{array}{l}\text { CM nonusers } \\
\text { days/year }\end{array}$} & \multirow{2}{*}{$\begin{array}{l}\text { CM users } \\
\text { days/year }\end{array}$} & \multicolumn{2}{|c|}{ Linear regression model } \\
\hline & & & $\overline{\beta(S E)^{\mathrm{a}}}$ & $P$-value \\
\hline Total, $\mathrm{N}^{\mathrm{b}}$ & 32710 & 11412 & & \\
\hline Antianemics ${ }^{c}$ & $7.7 \pm 39.3$ & $4.9 \pm 23.4$ & $-3.50(0.38)$ & $<0.0001$ \\
\hline Hemostatics & $4.1 \pm 24.1$ & $2.6 \pm 12.2$ & $-1.89(0.24)$ & $<0.0001$ \\
\hline Analgesics & $38.0 \pm 62.5$ & $38.0 \pm 50.3$ & $-0.42(0.64)$ & 0.5145 \\
\hline hormone-related agents & $16.4 \pm 57.3$ & $13.9 \pm 39.7$ & $-3.13(0.58)$ & $<0.0001$ \\
\hline Iron-deficient anemia, $\mathrm{n}^{\mathrm{b}}$ & 5259 & 2213 & & \\
\hline Antianemics & $30.9 \pm 73.5$ & $19.1 \pm 43.4$ & $-11.29(1.68)$ & $<0.0001$ \\
\hline Hemostatics & $8.3 \pm 34.6$ & $5.2 \pm 19.3$ & $-3.36(0.79)$ & $<0.0001$ \\
\hline Analgesics & $44.8 \pm 70.2$ & $42.8 \pm 57.4$ & $-2.24(1.69)$ & 0.1849 \\
\hline hormone-related agents & $14.2 \pm 40.6$ & $14.0 \pm 31.5$ & $-1.20(1.27)$ & 0.34 \\
\hline Hypermenorrhea, $n$ & 8388 & 3650 & & \\
\hline Antianemics & $13.2 \pm 49.6$ & $8.5 \pm 28.8$ & $-5.17(0.85)$ & $<0.0001$ \\
\hline Hemostatics & $7.9 \pm 34.2$ & $4.0 \pm 13.5$ & $-4.01(0.59)$ & $<0.0001$ \\
\hline Analgesics & $41.7 \pm 63.3$ & $38.9 \pm 47.4$ & $-3.51(1.16)$ & 0.0026 \\
\hline hormone-related agents & $24.0 \pm 71.1$ & $16.1 \pm 39.0$ & $-7.84(1.26)$ & $<0.0001$ \\
\hline Dysmenorrhea, $\mathrm{n}$ & 7456 & 4246 & & \\
\hline Antianemics & $7.9 \pm 37.4$ & $5.7 \pm 24.1$ & $-2.81(0.62)$ & $<0.0001$ \\
\hline Hemostatics & $4.8 \pm 24.8$ & $2.8 \pm 11.8$ & $-2.13(0.40)$ & $<0.0001$ \\
\hline Analgesics & $41.0 \pm 61.8$ & $37.2 \pm 47.0$ & $-3.46(1.07)$ & 0.0013 \\
\hline hormone-related agents & $17.1 \pm 56.2$ & $12.5 \pm 33.1$ & $-4.96(0.94)$ & $<0.0001$ \\
\hline
\end{tabular}

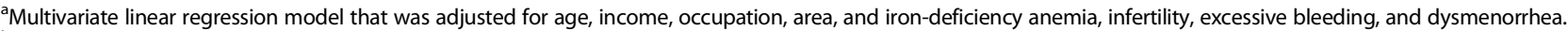
${ }^{\mathrm{b}} \mathrm{N}$, subject number in a group; and $\mathrm{n}$, subject number in a subgroup.

'Antianemics, including blood derivatives and iron; hemostatics, including coagulants and hemostatics; and analgesics, including non-steroid anti-inflammatory drugs and analgesics.

and that of hormone-related agents enlarged in patients with hypermenorrhea (-7.84 days/year/patient, $P<0.0001)$. Moreover, the consumption of analgesics became significantly different between CM users and nonusers in fibroid patients with dysmenorrhea (-3.46 days/year/ patient, $P=0.0013)$.

\section{Uterine fibroid-related medical cost between CM users and $\mathrm{CM}$ nonusers}

The mean total medical cost of CM users was 503.9 USD, and that of nonusers was 6294 USD per patients. After adjusting for age, income, occupation, area, iron-deficiency anemia, infertility, hypermenorrhea, and dysmenorrhea, the total annual medical cost for treating $\mathrm{CM}$ users was 0.45 -fold as much as that for treating $\mathrm{CM}$ nonusers (Table 4). Among the total medical cost, although the annual cost of Chinese medicine was 16.9 USD higher for CM users than that for CM nonusers, the annual cost of OPD $(-13$ USD/year, $P<0.0001)$, IPD ( -5611 USD/year, $P<0.0001)$, and ER $(-2.23$ USD/ year, $P<0.0001)$ for $C M$ users were lower than that for $\mathrm{CM}$ nonusers.

\section{Discussion}

$\mathrm{CM}$ is used by patients with uterine fibroids as an alternative to conventional western medicine. This study compared the consumption of conventional western medicine and the exact medical cost between $\mathrm{CM}$ users and nonusers in fibroid patients. Although more CM users were co-morbid with iron-deficiency anemia, hypermenorrhea, dysmenorrhea, and infertility, they consumed less fibroidrelated western medicines than $\mathrm{CM}$ nonusers. The difference in western medicine consumption between the two groups enlarged in patients with co-morbidities. Moreover, the utilization of CM did generate an extra medical cost, however, the total NHI-reimbursed annual health care fee per patient in CM users was substantially lower than that in $\mathrm{CM}$ nonusers.

Social-demographic trends regarding $\mathrm{CM}$ users in this study, which were extracted from a population-based database from 1996 to 2010, found that CM users in fibroid patients were more likely to be younger and white collar than nonusers. These trends was coincident with a previous study that extract patients from 2000 to 2003 [13], and also with studies on female-specific diseases, 
Table 4 Uterine fibroid-related cost in CM-users and nonusers

\begin{tabular}{|c|c|c|c|c|}
\hline \multirow[b]{2}{*}{ Type $^{b}$} & \multicolumn{2}{|c|}{ Cost, US\$/patient/year } & \multicolumn{2}{|c|}{ Linear regression model ${ }^{a}$} \\
\hline & CM nonusers & CM users & $\beta$ (SE) & $P$ value \\
\hline Total cost & $6294 \pm 41366$ & $503.9 \pm 4946$ & $0.45(1.05)$ & $<0.0001$ \\
\hline OPD & $16.9 \pm 146.5$ & $4.57 \pm 28.8$ & $0.75(1.02)$ & $<0.0001$ \\
\hline IPD & $6274 \pm 41317$ & $480 \pm 4833$ & $0.26(1.07)$ & $<0.0001$ \\
\hline surgery & $2931 \pm 19328$ & $213 \pm 1980$ & $0.27(1.07)$ & $<0.0001$ \\
\hline other (medication and admission) & $3335 \pm 25854$ & $261.23 \pm 3233$ & $0.27(1.07)$ & $<0.0001$ \\
\hline ER & $2.111 \pm 164.2$ & $0.097 \pm 3.51$ & $0.60(1.44)$ & $<0.0001$ \\
\hline$C M$ & $0.26 \pm 7.37$ & $18.7 \pm 597.7$ & $2.67(1.17)$ & $<0.0001$ \\
\hline
\end{tabular}

${ }^{a}$ Multivariate linear regression model for nature log transformation of cost and model was adjusted for age, income, occupation, area, iron-deficiency anemia, infertility, excessive bleeding, and dysmenorrhea.

${ }^{\mathrm{b}} \mathrm{OPD}$, outpatients, IPD, inpatients, ER, emergency room, and CM, Chinese medicine.

including gynecological and breast cancer $[16,17]$ and endometriosis [18]. In terms of co-morbidities, including iron-deficiency anemia, hypermenorrhea, dysmenorrhea, and infertility, this study showed that fibroid patients with co-morbidities tended to use CM, implying severe patients with symptoms were more likely to seek for alternative treatments. Several studies have also reported that most alternative medicine users are with symptoms of chronic diseases and poor health status [19-21], and "suffering from disease" is considered one of an important factors that triggers patients to use CM [19].

Similar to those being associated to $\mathrm{CM}$, high frequencies of taking conventional western medicine were associated with co-morbidities of hypermenorrhea, iron-deficiency anemia, and dysmenorrhea were associated with. However, patients who were co-morbid with infertility consumed less conventional western medicine than patients who were not co-morbid with infertility. Other social-demographic factors that associated with high consumption of conventional western medicine included age higher than 40 , being blue collar, income less than $520 \mathrm{USD} /$ month, and registration of the insurance in Central and Southern Taiwan. In terms of using $\mathrm{CM}, \mathrm{CM}$ users were less likely to consume conventional medicine, indicating that $\mathrm{CM}$ users reduced the use of conventional western medicine when they chose $\mathrm{CM}$ to treat diseases.

Antianemics, hemostatics, analgesics, and hormonerelated agents are commonly suggested medicines to control fibroid-related symptoms, i.e., iron-deficient anemia, hypermenorrhea, and dysmenorrhea [4]. As medications are only used in patients with well-defined signs or symptoms, among patients with a specific co-morbidity, the difference in the consumption of a co-morbidity-specific medicine implied that $\mathrm{CM}$ substituted traditional western medicine to treat this co-morbidity for $\mathrm{CM}$ users. For example, among patients co-morbid with hypermenorrhea, the low consumption of hemostatics in CM users implied CM substituted hemostatics to control symptoms of hypermenorrhea. Our data implied that $\mathrm{CM}$ might be useful to control symptoms caused by uterine fibroid. Animal studies and clinical studies have reported that CM might have two beneficial effects on uterine fibroid. First, $\mathrm{CM}$ is able to treat the symptoms related to uterine fibroid. Second, CM is able to reduce the size of fibroids. An animal study has shown that Shao-fu-zhu-yu decoction, a commonly used CM formula for dysmenorrhea, has been shown to relieve uterine contraction pain by its peripheral analgesic activities [22]. Another animal study reported that Bak-foong pills are able to directly downregulate mouse uterine tone [23]. However, clinical trials regarding the efficacy of $\mathrm{CM}$ on uterine fibroids are not of good quality. A systemic review reported that only two of 21 clinical trials are with strict randomization and blinding processes. These two rigorous randomized controlled trials reported that herbal preparation made fibroids to shrink, but the size reduction of fibroids by herbal preparation did not differ from the efficacy of mifepristone [24]. The present study provided epidemiological evidence showing difference in consumption of conventional western medicine between $\mathrm{CM}$ users and nonusers among fibroid patients, implying therapeutic effects to release symptoms caused by fibroids.

Complementary and alternative medicine is considered less expensive than conventional western medicine [11,12], and the mean cost per visit is lower for CM than for conventional western medicine [9]. However, since the use of $\mathrm{CM}$ generates a component of medical cost, whether using $\mathrm{CM}$ decreases the total medical cost is controversial. A review article concludes that there is still a lack of costeffectiveness research in CM [25]. This study found that the use of CM did reduce total medical cost paid by nation's health-care system for uterine fibroid. Although there was an extra expenditure in CM (18.7 USD/year/patient) spent by $\mathrm{CM}$ users, this extra expenditure was much less than the expenditure in conventional western medicine in both of the groups. Moreover, as CM users reduced the 
use of western medicine, the total medical reimbursement from NHI for CM users was 5610 UDS/year/patient less in $\mathrm{CM}$ users than in nonusers. The costs of uterine fibroids for OPD, IPD, and ER in CM users were all lower than that in $\mathrm{CM}$ nonusers. The major difference in medical cost between $\mathrm{CM}$ users and nonusers was the in-patient cost. CM users saved more than 2,622 dollars in the cost of surgery and 2,987 dollars in the cost of hospital admission and inpatient medication per patient annually, indicating CM users underwent less surgery and less hospitalization than $\mathrm{CM}$ nonusers. The present group has reported that $\mathrm{CM}$ users were more unlikely to under uterine surgery, including hysterectomy and myomectomy, with a hazard ratio of 0.18 [13]. This study confirmed that the decreased surgery rate in CM users reduced health care spending in uterine fibroid.

Using a database from the NHI, which is a governmentrun, single-payer national health insurance program that insures over $97 \%$ of citizens and over $99 \%$ of health-care institutes [26,27], this study provided epidemiological evidence showing different amount of conventional western medicine consumption and different medical cost between $\mathrm{CM}$ users and nonusers among patients with uterine fibroids. However, the first limitation of this study was that we could not verify whether patients underwent other forms of alternative medicines that are not covered by NHI, although the proportional of patients who took those alternative medicines was presumed small because of their high prices. The second limitation of this retrospective study was that it could not directly provide evidence for the efficacy of CM, but could only find a correlation between using $\mathrm{CM}$ and low consumption of western medicine and low cost. This correlation could also possibly come from the difference in healthcare seeking behavior between CM users and nonusers. Nevertheless, whatever the underlying reason was, this study highlighted that CM was associated with low consumption of conventional western medicine and low medical cost in fibroid patients. For practitioners, this study provided evidence that CM might be a potential therapeutic substitute for conventional western medicines to treat several symptoms of uterine fibroids. Moreover, for insurance policy makers, this study suggested that CM might be a proper area for health insurance to cover in fibroid patients.

\section{Conclusion}

This study revealed that CM reduced the consumption of conventional medicine, including total fibroid-related conventional medicine and three categories of conventional medicines (antianemics, hemostatics, and hormone-related agents) that are commonly used to release symptoms of fibroids. Moreover, although using CM increased a small amount of medical cost, the total medical cost for treating fibroid was less in CM users than in nonusers. Our results implied that CM might be a potential therapeutic substitute for conventional medicines to treat uterine fibroids with low cost.

\section{Abbreviations}

CM: Chinese medicine; IPD: Inpatient department; ER: Emergency room; ICD-9-CM: International Classification of Diseases, $9^{\text {th }}$ Revision, Clinical Modification; LHID2000: Longitudinal Health Insurance Database 2000; NHI: National Health Insurance; NSAID: Non-steroid anti-inflammatory drugs; OPD: Outpatient department; USD: US dollar.

\section{Competing interests}

The authors declare that they have no competing interests.

\section{Authors' contributions}

SYS designed the study and wrote the manuscript. CHM carried out all of the statistical analyses. DEM refined the manuscript and performed English editing. All authors read and approved the final manuscript.

\section{Acknowledgements}

This study was supported by grants from Taiwan Department of Health Clinical Trial and Research Center and for Excellence (MOHW104-TDU-B-212113002), and Taiwan Department of Health Cancer Research Center for Excellence (DOH102-TD-C-111-005).

\section{Author details}

'Department of Chinese Medicine, China Medical University Hospital, No. 2 Yude road, Taichung 40447, Taiwan. ${ }^{2}$ School of Post-baccalaureate Chinese Medicine, College of Chinese Medicine, China Medical University, Taichung 40402, Taiwan. ${ }^{3}$ College of Medicine, China Medical University, Taichung 40402, Taiwan. ${ }^{4}$ Management Office for Health Data, China Medical University Hospital, Taichung 40402, Taiwan. ${ }^{5}$ Department of Community Health Sciences, UCLA Fielding School of Public Health, Los Angeles, CA 90095-1772, USA.

Received: 28 October 2014 Accepted: 13 April 2015

Published online: 23 April 2015

\section{References}

1. Marino JL, Eskenazi B, Warner M, Samuels S, Vercellini P, Gavoni N, et al. Uterine leiomyoma and menstrual cycle characteristics in a population-based cohort study. Hum Reprod. 2004;19(10):2350-5.

2. Levy G, Hill MJ, Beall S, Zarek SM, Segars JH, Catherino WH. Leiomyoma: genetics, assisted reproduction, pregnancy and therapeutic advances. J Assist Reprod Genet. 2012;29(8):703-12.

3. Chalermchockchareonkit A, Tekasakul P, Chaisilwattana P, Sirimai K, Wahab N. Laparoscopic hysterectomy versus abdominal hysterectomy for severe pelvic endometriosis. Int J Gynaecol Obstet. 2012;116(2):109-11.

4. Marret H, Fritel X, Ouldamer L, Bendifallah S, Brun JL, De Jesus I, et al. Therapeutic management of uterine fibroid tumors: updated French guidelines. Eur J Obstet Gynecol Reprod Biol. 2012;165(2):156-64.

5. Cardozo ER, Clark AD, Banks NK, Henne MB, Stegmann BJ, Segars JH. The estimated annual cost of uterine leiomyomata in the United States. Am J Obstet Gynecol. 2012;206(3):211 e211-219.

6. Sabry M, Al-Hendy A. Innovative oral treatments of uterine leiomyoma. Obstet Gynecol Int. 2012;2012:943635.

7. Fernandez H, Farrugia M, Jones SE, Mauskopf JA, Oppelt P, Subramanian D. Rate, type, and cost of invasive interventions for uterine myomas in Germany, France, and England. J Minim Invasive Gynecol. 2009;16(1):40-6.

8. Chang LC, Huang N, Chou YJ, Lee CH, Kao FY, Huang YT. Utilization patterns of Chinese medicine and Western medicine under the National Health Insurance Program in Taiwan, a population-based study from 1997 to 2003. BMC Health Serv Res. 2008;8:170.

9. Tu CC, Li CS, Liu CM, Liu CC. Comparative use of biomedicine and Chinese medicine in Taiwan: using the NHI Research Database. J Altern Complement Med. 2011;17(4):339-46.

10. Shih CC, Liao CC, Su YC, Tsai CC, Lin JG. Gender Differences in Traditional Chinese Medicine Use among Adults in Taiwan. PLoS One. 2012;7(4):e32540.

11. Maxion-Bergemann S, Wolf M, Bornhoft G, Matthiessen PF, Wolf U. Complementary and alternative medicine costs - a systematic literature review. Forsch Komplementmed. 2006;13 Suppl 2:42-5. 
12. Hoenders HJ, Willgeroth FC, Appelo MT. Western and alternative medicine: a comparison of paradigms and methods. J Altern Complement Med. 2008;14(8):894-6.

13. Su SY, Muo CH, Morisky DE. Use of chinese medicine and subsequent surgery in women with uterine fibroid: a retrospective cohort study. Evid Based Complement Alternat Med. 2012;2012:617918.

14. Wye L, Sharp D, Shaw A. The impact of NHS based primary care complementary therapy services on health outcomes and NHS costs: a review of service audits and evaluations. BMC Complement Altern Med. 2009;9:5.

15. Hsieh SC, Lai JN, Lee CF, Hu FC, Tseng WL, Wang JD. The prescribing of Chinese herbal products in Taiwan: a cross-sectional analysis of the national health insurance reimbursement database. Pharmacoepidemiol Drug Saf. 2008;17(6):609-19.

16. Lin YH, Chiu JH. Use of Chinese medicine by women with breast cancer: a nationwide cross-sectional study in Taiwan. Complement Ther Med. 2011;19(3):137-43.

17. Navo MA, Phan J, Vaughan C, Palmer JL, Michaud L, Jones $K L$, et al. An assessment of the utilization of complementary and alternative medication in women with gynecologic or breast malignancies. J Clin Oncol. 2004;22(4):671-7

18. Fang RC, Tsai YT, Lai JN, Yeh CH, Wu CT. The traditional chinese medicine prescription pattern of endometriosis patients in taiwan: a population-based study. Evid Based Complement Alternat Med. 2012;2012:591391.

19. Liao HL, Ma TC, Chiu YL, Chen JT, Chang YS. Factors influencing the purchasing behavior of TCM outpatients in Taiwan. J Altern Complement Med. 2008;14(6):741-8.

20. Hwang JH, Han DW, Yoo EK, Kim WY. The utilisation of Complementary and Alternative Medicine (CAM) among ethnic minorities in South Korea. BMC Complement Altern Med. 2014;14:103.

21. Bahar Z, Kizilci S, Beser A, Besen DB, Gordes N, Ersin F, et al. Herbal therapies used by hypertensive patients in Turkey. Afr J Tradit Complement Altern Med. 2013;10(2):292-8.

22. Ma H, Su S, Duan J, Tang Y, Zhou J, Guo J, et al. Evaluation of the analgesic activities of the crude aqueous extract and fractions of Shao Fu Zhu Yu decoction. Pharm Biol. 2011;49(2):137-45.

23. Rowlands DK, Cui YG, Wong HY, Gou YL, Chan HC. Traditional Chinese medicine Bak Foong Pills alters uterine quiescence - possible role in alleviation of dysmenorrhoeal symptoms. Cell Biol Int. 2009;33(12):1207-11.

24. Liu JP, Yang H, Xia Y, Cardini F. Herbal preparations for uterine fibroids. Cochrane Database Syst Rev. 2009;2:CD005292.

25. Zhang F, Kong LL, Zhang YY, Li SC. Evaluation of impact on health-related quality of life and cost effectiveness of Traditional Chinese Medicine: a systematic review of randomized clinical trials. J Altern Complement Med. 2012;18(12):1108-20.

26. Lin MH, Chou MY, Liang CK, Peng LN, Chen LK. Population aging and its impacts: strategies of the health-care system in Taipei. Ageing Res Rev. 2010;9 Suppl 1:S23-7.

27. Chi C, Lee JL, Tsai SL, Chen WY. Out-of-pocket payment for medical care under Taiwan's National Health Insurance system. Health Econ. 2008;17(8):961-75.

\section{Submit your next manuscript to BioMed Central and take full advantage of:}

- Convenient online submission

- Thorough peer review

- No space constraints or color figure charges

- Immediate publication on acceptance

- Inclusion in PubMed, CAS, Scopus and Google Scholar

- Research which is freely available for redistribution 